\title{
Dopamine Modulates Inwardly Rectifying Potassium Currents in Medial Prefrontal Cortex Pyramidal Neurons
}

\author{
Yan Dong, ${ }^{2}$ Donald Cooper, ${ }^{1}$ Fernando Nasif, ${ }^{1}$ Xiu-Ti Hu, ${ }^{1}$ and Francis J. White ${ }^{1}$ \\ Departments of ${ }^{1}$ Cellular and Molecular Pharmacology and ${ }^{2}$ Neuroscience, Finch University of Health Sciences/The Chicago Medical School, North Chicago, \\ Illinois 60064
}

\begin{abstract}
Dopamine (DA) modulation of excitability in medial prefrontal cortex (mPFC) pyramidal neurons has attracted considerable attention because of the involvement of mPFC DA in several neuronal disorders. Here, we focused on DA modulation of inwardly rectifying $\mathrm{K}^{+}$ current (IRKC) in pyramidal neurons acutely dissociated from rat mPFC. $\mathrm{A} \mathrm{Cs}^{+}$-sensitive whole-cell IRKC was elicited by hyperpolarizing voltage steps from a holding potential of $-50 \mathrm{mV}$. DA $(20 \mu \mathrm{M})$ reduced IRKC amplitude, as did selective stimulation of DA $\mathrm{D}_{1}$ or $\mathrm{D}_{2}$ class receptors $\left(D_{1} R s\right.$ and $\left.D_{2} R s\right)$. $D_{1}$ Rs activate, whereas $D_{2}$ Rs inhibit, the adenylyl cyclase-cAMP-protein kinase A (PKA) signaling pathway. Suppression of IRKC by $D_{2} R$ stimulation was attributable to decreased PKA activity because similar inhibition was observed with PKA inhibitors, whereas enhancing PKA activity increased IRKC. This suggests that the DA $D_{1} R$ suppression of IRKC occurred through a PKA phosphorylation-independent process. Using outside-out patches of MPFC pyramidal neurons, which preclude involvement of cytosolic signaling molecules, we observed a Cs ${ }^{+}$-sensitive macroscopic IRKC that was suppressed by the membrane-permeable cyclic nucleotide Sp-cAMP but was unaffected by non-nucleotide modulators of PKA, suggesting direct interactions of the cyclic nucleotides with IRK channels. Our results indicate that DA suppresses IRKC through two mechanisms: $D_{1} R$ activation of cAMP and direct interactions of the nucleotide with IRK channels and $\mathrm{D}_{2} \mathrm{R}$-mediated dephosphorylation of IRK channels. The DA modulation of IRKC indicates that ambient DA would tend to increase responsiveness to excitatory inputs when PFC neurons are near the resting membrane potential and may provide a mechanism by which DA impacts higher cognitive function.
\end{abstract}

Key words: dopamine; $\mathrm{D}_{1}$ receptor; potassium; inward rectifier; prefrontal cortex; drug addiction

\section{Introduction}

Within the prefrontal cortex (PFC) dopamine (DA) modulates a variety of higher-order behavioral and cognitive processes, such as attention and working memory (for review, see Fuster, 2001; Miller and Cohen, 2001), and has been implicated in several neuronal disorders, including schizophrenia and drug addiction (for review, see Knable and Weinberger, 1997; Yang et al., 1999; Robbins and Everitt, 2002; Volkow et al., 2002). Given the critical role of DA in this cortical region, considerable effort has focused on the cellular mechanisms by which DA modulates the function of PFC neurons. However, a consensus on precise DA actions has been slow to unfold despite over two decades of effort. Historically, the effects of DA on deep-layer PFC pyramidal neurons (V

Received 0ct. 18, 2003; revised Jan. 20, 2004; accepted Feb. 9, 2004

This work was supported by United States Public Health Service Grant DA12618 from the National Institute on Drug Abuse (NIDA). F.J.W. is a recipient of NIDA Senior Scientist Award DA00456. We thank Kerstin Ford and Lori Baker for excellent technical assistance, Drs. D. J. Surmeier, Robert Foehring, and P. Couceyro for comments about our results, and Dr. Bill Ju for plotting Figure 8 .

Correspondence should be addressed to either Dr. Xiu-Ti Hu or Dr. Francis J. White, Department of Cellular and Molecular Pharmacology, Finch University of Health Sciences/The Chicago Medical School, 3333 Green Bay Road, North Chicago, IL 60064. E-mail: hux@finchcms.edu or francis.white@finchcms.edu.

Y. Dong's present address: Department of Psychiatry and Behavioral Sciences, Stanford University School of Medicine, Palo Alto, CA 94304. E-mail: yandong@stanford.edu.

D. Cooper's present address: Department of Psychiatry, University of Texas, Southwestern Medical Center, Dallas, TX 75390-9070. E-mail: d-cooper2@northwestern.edu.

DOI:10.1523/JNEUROSCI.4715-03.2004

Copyright $\odot 2004$ Society for Neuroscience $\quad$ 0270-6474/04/243077-09\$15.00/0 and VI) have been described as inhibitory or excitatory on the basis of either extracellular or intracellular recordings conducted in vivo as well as in vitro (for review, see Yang et al., 1999). Only recently have investigators turned their attention to the mechanisms by which $D A$, acting through the five known $D A\left(D_{1}-D_{5}\right)$ receptors, modulates voltage-gated conductances that determine neuronal excitability. Identifying the coordinated responses of these conductances to DA receptor stimulation is essential for a thorough understanding of how DA modulates neuronal activity in the PFC.

One group of voltage-gated conductances that has received attention with respect to DA modulation in the PFC is the voltage-gated $\mathrm{K}^{+}$currents (VGKCs). It has been suggested that $\mathrm{D}_{1} \mathrm{R}$ stimulation suppresses a slowly inactivating outward $\mathrm{K}^{+}$ conductance in PFC pyramidal neurons (Yang and Seamans, 1996; Gorelova and Yang, 2000). Using acutely dissociated medial PFC (mPFC) neurons, which allows excellent voltage con-

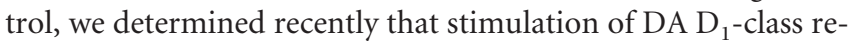
ceptors $\left(D_{1} R s\right)$ selectively suppresses a slowly inactivating VGKC component $\left(I_{D}\right)$ in $\mathrm{mPFC}$ neurons without altering the rapidly inactivating (A-type) current or a very slowly inactivating $\mathrm{K}^{+}$ current $\left(I_{\mathrm{K}}\right)$ (Dong and White, 2003). DA $\mathrm{D}_{2}$-class $\left(\mathrm{D}_{2} / \mathrm{D}_{3} / \mathrm{D}_{4}\right)$ receptor $\left(\mathrm{D}_{2} \mathrm{R}\right)$ activation did not modulate these voltage-gated $\mathrm{K}^{+}$currents (Dong and White, 2003). Here, we continue our investigations of DA modulation of $\mathrm{K}^{+}$conductance in $\mathrm{mPFC}$ neurons by focusing on inwardly rectifying $\mathrm{K}^{+}$current (IRKC). 
IRKCs are critical for setting resting membrane potential, shaping action potentials, and balancing the intracellular $\mathrm{K}^{+}$/ $\mathrm{Na}^{+}$equilibrium. Thereby, IRKC is an essential determinant of neuronal excitability (Hille, 2001). At least seven subfamilies of inward rectifier $\mathrm{K}^{+}$(Kir) channels have been cloned (Kir 1.0-Kir 7.0) (for review, see Jan and Jan, 1997; Nichols and Lopatin, 1997; Coetzee et al., 1999), most of which are expressed in cortical deep layers (V and VI) (Karschin et al., 1996). Modulation of this conductance by DA in mPFC neurons may provide a mechanism by which DA impacts higher cognitive functions. Accordingly, we sought to characterize IRKC in MPFC pyramidal neurons and to identify potential modulation of IRKC by $\mathrm{D}_{1}$ Rs and $\mathrm{D}_{2}$ Rs.

\section{Materials and Methods}

Acute dissociation. Deep layer (V and VI) mPFC pyramidal neurons from 4-to 5-week-old Sprague Dawley rats were acutely dissociated using protocols described previously (Dong and White, 2003). In brief, rats were anesthetized with methoxyflurane (Mallinckrodt, Mundelein, IL) and decapitated. Brains were quickly removed, blocked, and sliced on a DSK microslicer (Campden Instruments, Lafayette, IN) in a $1-2^{\circ} \mathrm{C}$ sucrose solution containing the following (in $\mathrm{mm}$ ): 234 sucrose, $2.5 \mathrm{KCl}, 1$ $\mathrm{Na}_{2} \mathrm{HPO}_{4}, 11$ glucose, $4 \mathrm{MgSO}_{4}, 0.1 \mathrm{CaCl}_{2}$, and 15 HEPES, pH 7.35 (300 $\mathrm{mOsm} / \mathrm{l})$. Coronal slices $(400 \mu \mathrm{m})$ were incubated $1-4 \mathrm{hr}$ at room temperature in a sodium bicarbonate-buffered Earle's balanced salt solution bubbled with $95 \% \mathrm{O}_{2}-5 \% \mathrm{CO}_{2}$ and containing the following (in $\mathrm{mm}$ ): 1 kynurenic acid, 1 pyruvic acid, $0.1 \mathrm{~N}$-nitroarginine, and 0.005 glutathione, pH 7.4 (300 mOsm/l). Individual slices were then placed in a $\mathrm{Ca}^{2+}$ free buffer [in mM: $140 \mathrm{Na}$-isethionate, $2 \mathrm{KCl}, 4 \mathrm{MgCl}_{2}, 23$ glucose, and 15 HEPES, pH 7.4 (300 mOsm/l)], and, under a dissecting microscope, the mPFC was isolated. The mPFC tissue was then placed into an oxygenated, HEPES-buffered HBSS containing $1.5 \mathrm{mg} / \mathrm{ml}$ protease (type $\mathrm{XIV}$ ) at $35^{\circ} \mathrm{C}$ for $30 \mathrm{~min}$. The enzyme chamber also contained the following (in mM): 1 kynurenic acid, 1 pyruvic acid, $0.1 \mathrm{~N}$-nitroarginine, and 0.005 glutathione, $\mathrm{pH} 7.4$ (300 mOsm/l). Unless otherwise stated, all chemicals were obtained from Sigma (St. Louis, MO). After enzymatic treatment, the tissue was rinsed several times in the $\mathrm{Ca}^{2+}$-free buffer and triturated with a graded series of fire-polished Pasteur pipettes. The cell suspension was placed in a $35 \mathrm{~mm}$ Lux Petri dish (Nunc, Naperville, IL), which was mounted on an inverted microscope. Cells were then given several minutes to settle down before electrophysiological recording.

Whole-cell recordings. Electrodes were pulled from Corning (Corning, NY) 7052 glass (Flaming/Brown P-97 puller; Sutter Instruments, Novato, CA) and fire-polished (MF-83 microforge; Narishige, Hempstead, $\mathrm{NY}$ ) just before use. The intracellular recording solution for recording IRKCs was as follows (in mM): $70 \mathrm{~K}_{2} \mathrm{SO}_{4}, 60 \mathrm{~N}$-methyl-glucamine, 30 HEPES, 5 BAPTA, 12 phosphocreatine, $3 \mathrm{Na}_{2}$ ATP, $0.2 \mathrm{Na}_{3} \mathrm{GTP}, 2 \mathrm{MgCl}_{2}$, and $0.5 \mathrm{CaCl}_{2}, \mathrm{pH} 7.2(275 \mathrm{mOsm} / \mathrm{l})$. The normal extracellular recording solution contained the following (in $\mathrm{mm}$ ): $120 \mathrm{Na}$-isethionate, 10 HEPES, 12 glucose, 17.5 sucrose, $1-20 \mathrm{KCl}, 4 \mathrm{MgCl}_{2}$, and $0.001 \mathrm{TTX}, \mathrm{pH}$ 7.35 (300 mOsm/l). Extracellular recording solutions were applied via one of a series of four glass capillaries $(\sim 250 \mu \mathrm{m}$ inner diameter $)$ in which gravity-fed flow was regulated by electronic valves (Bio-Logic, Claix, France). Recordings were obtained with an Axon Instruments (Foster City, CA) 200A patch-clamp amplifier, controlled, and monitored with a Pentium personal computer running pClamp (version 8.1) with a $125 \mathrm{kHz}$ interface (Axon Instruments). Electrode resistances were $\sim 1-4 \mathrm{M} \Omega$ in bath. After formation of the gigaohm seal and subsequent cell rupture, series resistance was compensated (70-80\%) and periodically monitored. Recordings were restricted to neurons with pyramidal soma and small remnants of the apical dendrites. The average whole-cell capacitance $(\sim 12 \mathrm{pF})$ of the recorded neurons was consistent with our previous study of acutely dissociated mPFC neurons (Dong and White, 2003). Series resistance was steadily below $8 \mathrm{M} \Omega(<10 \%)$. Recordings were performed at room temperature $\left(22-24^{\circ} \mathrm{C}\right)$. The liquid junction potential $(\sim 2 \mathrm{mV})$ was not compensated. In the experiments in which the extracellular $\mathrm{K}^{+}$was sharply changed, we used $\mathrm{Cs}^{+}$to block the IRKC and calculate the $\mathrm{Cs}^{+}$-sensitive IRKC by subtraction, therefore minimizing the impact of junction potential.
Outside-out recordings from acutely dissociated PFC neurons. Outsideout voltage-clamp patch recordings were used to measure the macroscopic IRKC. Briefly, the electrodes were intentionally made larger (500 $\mathrm{k} \Omega$ ) than whole-cell electrodes $(2-6 \mathrm{M} \Omega)$. After the whole-cell configuration was established, the electrode was slowly pulled away from the cell. The membrane capacitance was used as an indicator and simultaneously monitored. The outside-out patch was determined to be successfully established when the capacitance significantly dropped with no change in the gigaohm seal. On some occasions, when cells did not firmly stick to the bottom of the dish and moved with the recording electrode, another electrode was used to block the cell. The internal solutions for outsideout patch recordings were identical to those in the whole-cell recordings. All drugs studied with this preparation were applied through the bath solution.

Drugs and drug application. All reagents were obtained from Sigma except ATP and GTP (Boehringer Mannheim, Indianapolis, IN) and protein kinase inhibitor (PKI), SKF 81297, SKF 38393, quipirole, eticlopride, SCH 23390, BAPTA, okadaic acid, cBIMP, Sp-8-bromo-cAMP, Sp-cGMP, Rp-cAMP, and H8 (Calbiochem, La Jolla, CA). PKI was added in the internal solution, and PKI effects were observed when the internal solution diffused into the cell after membrane rupture. All other drugs were bath applied. In recordings of dissociated neurons, the recorded neuron was locally and continuously perfused by external solution delivered from one of the four series capillaries (bath feeding system; BioLogic). Drugs were applied to the recorded neuron by switching to a capillary that delivered the pertinent drug-containing bath. Using this bath exchange system, the initiation and termination of drug perfusion could be completed within $500 \mathrm{msec}$. Drug that were used to directly inhibit or stimulate cytosolic signaling molecules are membrane permeable.

Data analysis. Dose-response data were fit with a Langmuir isotherm of the following form: $C /\left(C+\mathrm{IC}_{50}\right)$, where $C$ is the concentration of blocking agent. Statistica (StatSoft, Tulsa, OK) was used for most of the statistical analysis. Origin (Microcal, Northampton, MA) was used to plot the current traces and graphs. Box-whisker plots were used to show most data because of small sample sizes. The box plot presented the distribution as a box, with the median as a central line and the hinges as the edge of the box, which divided the upper and lower halves of the distribution in two. The inner fence, starting from the edge of the box, ran to the limits of the distribution, excluding outliers, which are defined as points that are two times the inter-quartile range beyond the inner fence. Outliers are shown as open circles. Given that the whole-cell IRKC mainly comprises the $\mathrm{Cs}^{+}$-sensitive IRKC, in the experiment testing the modulation of IRKC by DA receptors and intracellular signalings, wholecell current amplitude was regarded as the amplitude of IRKC and used for statistics. For the drug-induced effects, the averages of three traces before drug administration were compared with three traces during drug perfusion using a paired $t$ test.

\section{Results}

\section{Characterization of IRKC}

Pyramidal neurons within neocortex express VGKC attributable to Kv1.0-Kv4.0 family subunits (Rudy et al., 1992; Sheng et al., 1994; Wang et al., 1994; Serodio and Rudy, 1998), and these outward $\mathrm{K}^{+}$currents exhibit activation by depolarization starting at approximately $-45 \mathrm{mV}$ (Dong and White, 2003). Therefore, inward currents, evoked by holding the membrane potential at $-50 \mathrm{mV}$ and stepping to hyperpolarized potentials, are unlikely attributable to the currents carried by Kv family channels and are most probably attributable to IRK channels. By using such protocols, we were able to selectively study IRK currents $(n=11)$ (Fig. 1A1). These inward currents were pharmacologically consistent with IRKC in their $\mathrm{Cs}^{+}$sensitivity (Hille, 2001). Low concentrations of $\mathrm{Cs}^{+}(1 \mathrm{~mm})$ preferentially blocked the inward currents, leaving outward currents intact $(n=9)$ (Fig. $1 A 2)$. The $\mathrm{Cs}^{+}{ }^{-}$-sensitive IRKC was obtained in Figure $1 A 3$ by subtraction of traces in Figure $1 A 2$ from that in Figure $1 A 1$. The 


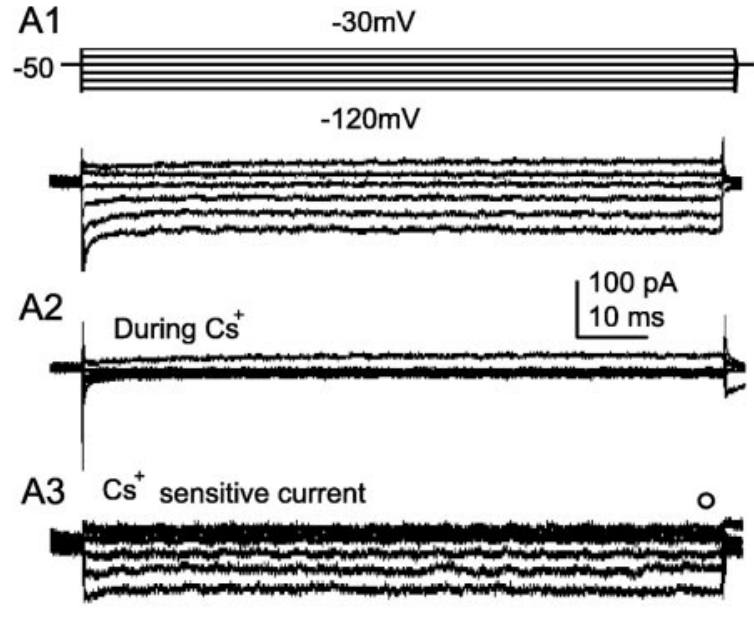

B

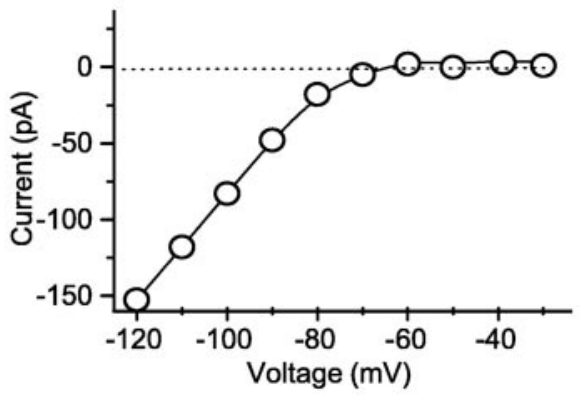

C1

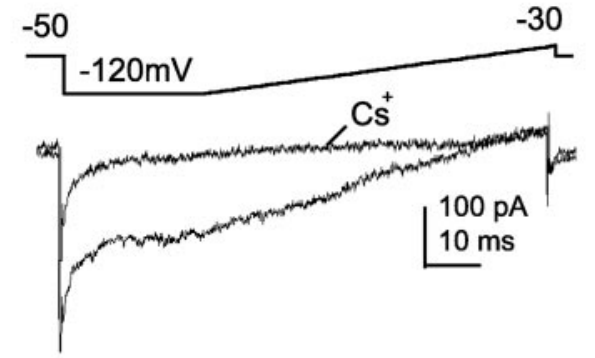

C2

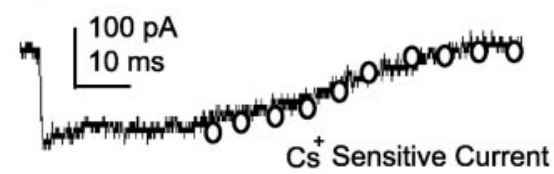

Figure 1. Whole-cell IRKC in mPFC pyramidal neurons. A1, The whole-cell IRKC was elicited when the membrane potential stepped from $-50 \mathrm{mV}$ to a $200 \mathrm{msec}$ voltage command (from -30 to $-120 \mathrm{mV}$ with $10 \mathrm{mV}$ increments). A2, The whole-cell IRKC was blocked by perfusion of $1 \mathrm{~mm} \mathrm{Cs}{ }^{+} . A 3$, The $C s^{+}$-sensitive IRKC was obtained after subtraction of the traces in $A 2$ from those in $A 1$. In $A 1-A 3$, every other trace of all traces elicited by the protocols were presented. $B$, The $\mathrm{I}-V$ curve of the $\mathrm{Cs}^{+}$-sensitive currents showed strong inward rectification. C1, The IRKC was elicited by a ramp protocol, comprising a 20 msec step to $-120 \mathrm{mV}$ and a $60 \mathrm{msec}$ ramp from -120 to $-30 \mathrm{mV}$. The trace elicited by the ramp protocol could be blocked by $\mathrm{Cs}^{+}(1$ $\mathrm{mm}) .\left(2\right.$, The $\mathrm{Cs}^{+}$-sensitive trace was obtained after subtraction. The first part of the current (by voltage step) is comparable with that elicited by the step protocol, as that in $A 3$. The second part (elicited by voltage ramp) is comparable with the $I-V$ curve obtained from step protocols, as in $B$. The open circles shown in the inset are data points from $B$ after adjustment of the time course with voltage change.

averaged amplitude of this $\mathrm{Cs}^{+}{ }^{+}$-sensitive current was $197 \pm 36$ $\mathrm{pA}$ at $-120 \mathrm{mV}\left(n=9\right.$; all examined cells exhibited $\mathrm{Cs}^{+}$-sensitive component). The voltage-current relationship of $\mathrm{Cs}^{+}$-sensitive currents displayed strong inward rectification (Fig. $1 B$ ). A voltage ramp was designed to rapidly generate IRKC, as well as a description of the $I-V$ relationship. In the ramp protocol, the 20 msec test step to $-120 \mathrm{mV}$ from the holding potential of $-50 \mathrm{mV}$ was followed by a ramp from -120 to $-30 \mathrm{mV}$ with a time course of $60 \mathrm{msec}$. The ramp current could also be blocked by a low ( 1 $\mathrm{mm}$ ) concentration of $\mathrm{Cs}^{+}$(Fig. 1C1). The $\mathrm{Cs}^{+}$-sensitive ramp current was isolated by subtraction (Fig. 1C2). The first part of the trace, which was elicited by the $20 \mathrm{msec}$ step to $-120 \mathrm{mV}$, was equivalent to the trace elicited by step protocols in Figure $1 A$. The trace evoked by ramps was equivalent to the $I-V$ curve obtained by steps in Figure $1 B$. In Figure 1C2, the open circles are the voltages from the $I-V$ curve generated from step protocols. They were consistent with the time points corresponding to the voltages obtained from the ramp protocols $(n=4)$.

IRKC could be blocked by a wide concentration range of $\mathrm{Cs}^{+}$ (0.01-10 mM) and was maximally blocked at low millimolar concentrations (Fig. $2 A$ ). This dose-dependent blockade is summarized in Figure $2 B$ ( $n=4$ for each data point $)$ and could be well fit with one Langmuir isotherm ( $\mathrm{IC}_{50}$ of $0.12 \mathrm{~mm}$ ). Results from this pharmacological study are consistent with previous reports in other types of neurons (Hille and Schwarz, 1978; Mermelstein et al., 1998). We next examined the ionic selectivity of the IRKC. Replacing extracellular $\mathrm{K}^{+}$with $\mathrm{Na}^{+}$significantly attenuated $\mathrm{Cs}^{+}$-sensitive IRKC $(n=3)$ (Fig. $\left.2 C\right)$, as has been observed in other types of neurons (Mermelstein et al., 1998). Another way to examine the ionic selectivity of IRKC is to measure the zerocurrent potential (reversal potential). The reversal potential of a specific type of ion channels is determined by concentration gradients and is theoretically calculated by the Nernst equation $\left(E_{\mathrm{rev}}=(R T / z F) \operatorname{Ln}\left([K]_{\mathrm{o}} /[K]_{\mathrm{i}}\right)\right.$. For example, if the intracellular $\left[\mathrm{K}^{+}\right]_{\mathrm{i}}$ is $140 \mathrm{mM}$ and the extracellular $\left[\mathrm{K}^{+}\right]_{\mathrm{o}}$ is $20 \mathrm{mM}$, the $E_{\text {rev }}$ should be $-50 \mathrm{mV}$. In our recordings, IRKC was altered, responding to the shift of extracellular $\mathrm{K}^{+}$from $20 \mathrm{mM}(n=7)$ to 7 $\mathrm{mM}(n=4)$ and $2.6 \mathrm{~mm}(n=4)$ (Fig. $2 D)$. The amplitude of inward currents decreased in response to the decrease of $\left[\mathrm{K}^{+}\right]_{\mathrm{o}}$. The observed reversal potential changed with alterations of $\left[\mathrm{K}^{+}\right]_{\mathrm{o}}$ in a manner consistent with the Nernst equation (Fig. $2 \mathrm{E}$ ).

\section{Dopamine modulation of IRKC}

Pyramidal neurons in mPFC express both DA $D_{1}$-class $\left(D_{1} / D_{5}\right)$ and $\mathrm{D}_{2}$-class $\left(\mathrm{D}_{2} / \mathrm{D}_{3} / \mathrm{D}_{4}\right)$ receptors with different laminar topographies (for review, see Goldman-Rakic, 1999a; Yang et al., 1999). The selective $\mathrm{D}_{1} \mathrm{R}$ agonist SKF $81297(0.1 \mu \mathrm{M})$ produced a reversible suppression of $\mathrm{Cs}^{+}$-sensitive IRKC that was prevented by the selective $\mathrm{D}_{1} \mathrm{R}$ antagonist $\mathrm{SCH} 23390(1 \mu \mathrm{M})$ (Fig. 3A). $\mathrm{D}_{1} \mathrm{R}$ stimulation suppressed IRKC amplitude in 9 of 10 recorded neurons ( $16 \pm 4 \% ; n=9 ; p<0.05$; one outlier was excluded from statistics) (Fig. 3D). One neuron displayed enhanced IRKC amplitude during $\mathrm{D}_{1} \mathrm{R}$ stimulation.

$\mathrm{D}_{1}$ Rs positively couple to the adenylyl cyclase-cAMP-protein kinase A (PKA) signaling pathway, whereas $\mathrm{D}_{2}$ Rs inhibit this pathway (for a recent review, see Hartman and Civelli, 1997). Given that $\mathrm{D}_{1}$ Rs suppressed IRKC amplitudes, we predicted that stimulation of $\mathrm{D}_{2}$ Rs might enhance IRKC. Surprisingly, selective stimulation of $\mathrm{D}_{2} \mathrm{Rs}$ with $0.1 \mu \mathrm{M}$ quinpirole also suppressed IRKC amplitude ( $15 \pm 5 \% ; n=9 ; p<0.05$; note that there were four nonresponders in these nine cells) (Fig. $3 D$ ). The mean suppression among the five responders was $26 \pm 4 \%$, and the suppression could be prevented by the $\mathrm{D}_{2} \mathrm{R}$ antagonist eticlopride ( 1 $\mu \mathrm{M})$ (Fig. $3 B)$. DA $(20 \mu \mathrm{M})$, the endogenous agonist for all DA receptors, induced a significant inhibition of IRKC that appeared additive to the effects of selective stimulation of the receptor families $(33 \pm 4 \%$; $n=5$; $p<0.05)$ (Fig. 3C,D).

Given that DA also inhibits whole-cell VGKC in mPFC pyramidal neurons (Dong and White, 2003), we next examined the roles of $\mathrm{D}_{1}$ Rs and $\mathrm{D}_{2}$ Rs in suppressing both types of $\mathrm{K}^{+}$currents. 
Using a protocol in which whole-cell IRKC and VGKC are sequentially elicited by a hyperpolarization step $(-120 \mathrm{mV}$, $400 \mathrm{msec}$ ) from a holding potential of -50 $\mathrm{mV}$ and a subsequent depolarization step (+30 mV, $300 \mathrm{msec}) . \mathrm{D}_{1} \mathrm{R}$ stimulation with SKF $81297(0.1 \mu \mathrm{M})$ induced a slight inhibition of IRKC amplitude (11 $\pm 3 \%)$ but a strong inhibition of VGKC (34 \pm $5 \% ; n=4$ ) (Fig. 4A1,B). Suppression of VGKC only affected the slow component of this current $\left(I_{D}\right)$, leaving the rapid inactivating component $\left(I_{A}\right)$ intact, as we observed previously (Dong and White, 2003). $\mathrm{D}_{2} \mathrm{R}$ stimulation with quinpirole $(0.1 \mu \mathrm{M})$ substantially suppressed IRKC amplitude ( $29 \pm 6 \% ; n=4$; all four examined cells are responsive to $\mathrm{D}_{2} \mathrm{R}$ stimulation), leaving VGKC intact (net change, $2 \pm 1 \% ; n=4)$. As expected, DA $(20 \mu \mathrm{M})$ inhibited both IRKC ( $31 \pm 4 \% ; n=3)$ and VGKC $(35 \pm 8 \% ; n=3)$. This result demonstrates the differential and dynamic modulation of $\mathrm{K}^{+}$current by DA in mPFC neurons, i.e., modulation of VGKC is primarily mediated by $\mathrm{D}_{1} \mathrm{Rs}$, whereas modulation of IRKC is preferentially mediated by $\mathrm{D}_{2}$ Rs. Note that the mean suppression of IRKC by $\mathrm{D}_{2} \mathrm{R}$ stimulation is $\sim 30 \%$ in this set of experiments (Fig. 4) but $\sim 15 \%$ in previous experiments (Fig.

3). The discrepancy may be attributable to the different protocols (steps vs ramps) but is more likely attributable to the small sample size. In the current experiment, four of four cells are responders, whereas in the previous experiment, five of nine are responders.

\section{Mechanism of DA-mediated modulation of IRKC}

Because $D_{1} R$ stimulation activates, whereas $D_{2} R$ s suppress, the adenylyl cyclase-cAMP-PKA signaling cascade, selective DA receptor class activation should modulate IRKC in opposite directions. However, our results indicate that stimulation of either $D_{1}$ Rs or $D_{2}$ Rs suppresses IRKC. To elucidate how $D_{1}$ Rs and $D_{2} R s$ converge to modulate IRKC in the same direction, we first examined how IRKC responds to different degrees of PKA-induced phosphorylation. Inactivation of PKA with $20 \mu \mathrm{M} \mathrm{H} 8$, a PKA blocker, suppressed IRKC amplitude ( $15 \pm 6 \%$; $n=5 ; p<0.05)$ (Fig. $5 A, E, F$ ), whereas stimulation of PKA with $25 \mu \mathrm{M}$ cBIMP, a potent membrane-permeable PKA activator, enhanced IRKC amplitude $(23 \pm 7 \% ; n=5 ; p<0.05)$ (Fig. $5 B, F)$. When a more specific PKA blocker, PKI $(1 \mathrm{U} / \mathrm{ml})$, was filled in the recording pipette and diffused into the cell, IRKC amplitude decreased $(n=$ 4 for each group) (Fig. 5C). Apparently, the basal phosphorylation state of IRK channels is determined by an equilibrium between constitutive activity of PKA and protein phosphatase (PP). Increasing basal phosphorylation of IRKC channels by blocking PP-induced dephosphorylation with okadaic acid $(10 \mu \mathrm{M})$ enhanced IRKC $(17 \pm 6 \% ; p<0.05 ; n=5)$ (Fig. $5 D, F)$. On the basis of these results, we conclude that PKA-induced phosphorylation enhances IRKC amplitude and IRKC exists under constitutive PKA-induced phosphorylation in mPFC neurons. This conclusion explains the $\mathrm{D}_{2} \mathrm{R}$-mediated inhibition of IRKC. Under such conditions, $\mathrm{D}_{1} \mathrm{R}$ stimulation, which increases PKA ac-
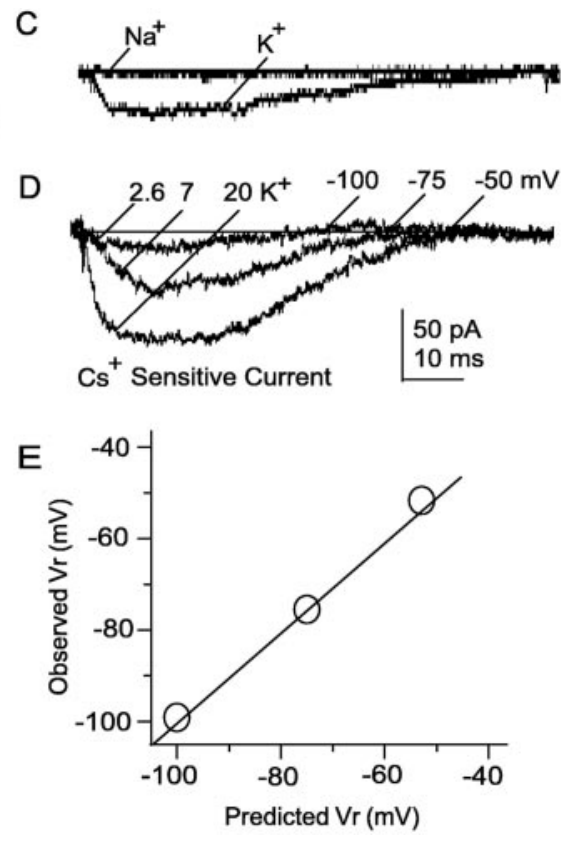

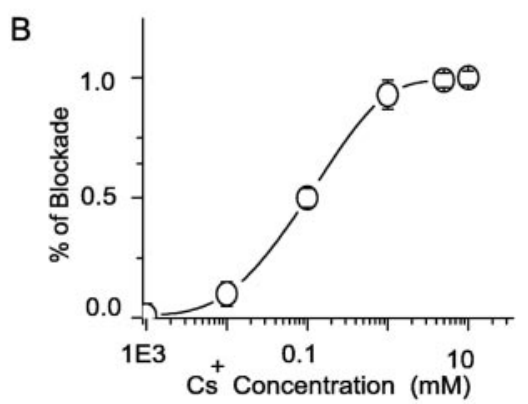

Figure 2. Characterization of IRKC. $A$, Extracellular application of $\mathrm{Cs}^{+}$blocked IRKC in a concentration-dependent manner. $B$ selectively conduct $\mathrm{K}^{+}$ions. When $\mathrm{K}^{+}$was replaced with $\mathrm{Na}^{+}{ }^{+}$in the bath, $\mathrm{no} \mathrm{Cs}^{+}$-sensitive IRKC was observed. 作 ( $n=4)$, respectively. $E$, The $V_{r}$ could be theoretically predicted with the Nernst equation. The observed $V_{r}$ was

tivity, should enhance rather than suppress IRKC. Then why did we observe an inhibition of IRKC during $\mathrm{D}_{1} \mathrm{R}$ stimulation? Perhaps there is a phosphorylation-independent pathway mediating $\mathrm{D}_{1} \mathrm{R}$ modulation of IRKC. If so, blockade of PKA activity should not occlude the $\mathrm{D}_{1} \mathrm{R}$-mediated effect. In fact, when PKA activity was blocked with $\mathrm{H} 8(20 \mu \mathrm{M})$, stimulation of $\mathrm{D}_{1}$ Rs with SKF $81297(0.1 \mu \mathrm{M})$ induced an additive inhibition of IRKC $(11 \pm$ $4 \% ; n=3$ ) (Fig. 5E), indicating the existence of a PKAindependent modulation of IRKC by $\mathrm{D}_{1}$ Rs.

If the adenylyl cyclase-cAMP-PKA cascade mediates both $D_{1} R$ and $D_{2} R$ modulation of IRKC, $D_{1} R$ signaling should diverge from $\mathrm{D}_{2} \mathrm{R}$ signaling from a position upstream of PKA. We therefore focused on cAMP. Perfusion with $100 \mu \mathrm{M}$ Sp-8-bromocAMP (a membrane-permeable cAMP analog and PKA stimulator) suppressed (suppression, $>15 \%$ ) IRKC in 5 of 14 recorded cells (Fig. 6A,C) but enhanced (enhancement, >15\%) IRKC amplitude in 5 of 14 cells (Fig. $6 B, C$ ); in the other four cells, the net change of IRKC amplitude was $<15 \%$. This observation suggests that increased intracellular cAMP, which would be triggered by $\mathrm{D}_{1} \mathrm{R}$ stimulation, can modulate IRKC in opposite directions. If so, why did $\mathrm{D}_{1} \mathrm{R}$ stimulation, which elevates intracellular cAMP levels, not result in the bidirectional modulation of IRKC? This contradiction was reconciled when we elevated the concentrations of SKF $81297(5 \mu \mathrm{M})$. This time, 14 of 28 neurons displayed reduced IRKC (reduction, $>15 \%$ ) in response to $\mathrm{D}_{1} \mathrm{R}$ stimulation, whereas 4 of 28 neurons showed enhancement (enhancement, $>15 \%$ ). The additional 10 neurons were not affected during perfusion of SKF 81297 (Fig. 6C). The bidirectional effect of $\mathrm{D}_{1} \mathrm{R}$ stimulation was also observed when another $\mathrm{D}_{1} \mathrm{R}$ agonist, SKF $38393(5 \mu \mathrm{M})$, was used. Suppression ( $>15 \%)$ of IRKC was observed in 7 of 20 recorded neurons, enhancement $(>15 \%)$ in five neurons, and no obvious alteration in the other eight neurons 

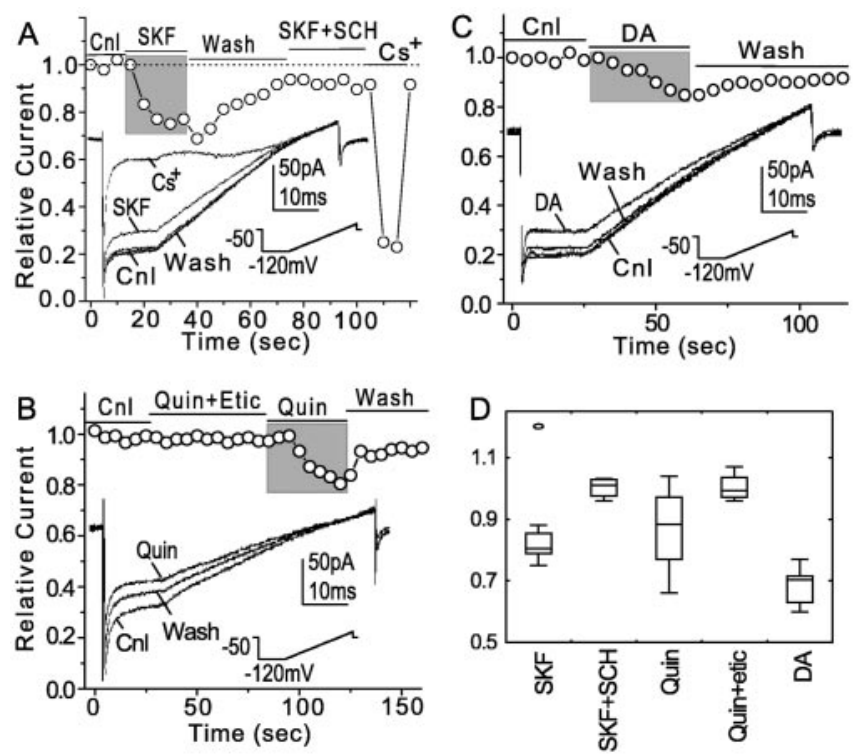

Figure 3. DA inhibits IRKC amplitude via both $D_{1}$ Rs and $D_{2} R s . A$, Activation of $D_{1} R$ by its agonist SKF 81297 (0.1 $\mu \mathrm{m}$; SKF) suppressed IRKC amplitude. The suppression was reversed by washout (Wash) and was prevented by the $\mathrm{D}_{1} \mathrm{R}$ antagonist SCH $23390(1 \mu \mathrm{m} ; \mathrm{SCH})$. The IRKC was almost fully blocked by $1 \mathrm{~mm} \mathrm{Cs}{ }^{+}$. B, Activation of the $D_{A} D_{2} R$ with quinpirole $(0.1 \mu \mathrm{m}$; Quin) also suppressed IRKC. The suppression was partially washable and was prevented by the $\mathrm{D}_{2}$ R antagonist eticlopride (1 $\mu \mathrm{m} ;$ Etic). C, Perfusion of DA (20 $\left.\mu \mathrm{m}\right)$ suppressed IRKC amplitude, an effect that was partially washable. $D$, The DA receptor-mediated suppression of IRKC is summarized. Activation of $D_{1}$ Rs with $0.1 \mu \mathrm{m}$ SKF 81297 suppresses $16 \pm 4 \%$ of IRKC ( $n=9$ of 10). One neuron showed an enhancement, indicated as the upper outlier. Relative IRKC amplitude during coperfusion of $0.1 \mu \mathrm{m}$ SKF 81297 and $1 \mu \mathrm{M} \mathrm{SCH} 23390$ was $98 \pm 2 \%(n=6)$. Stimulation of $D_{2} R s$ with $0.1 \mu \mathrm{m}$ quinpirole suppressed IRKC $15 \pm 5 \%(n=9$, among which, 4 displayed $<10 \%$ alterations). The relative IRKC amplitude was $98 \pm 2 \%(n=5)$. Perfusion of $20 \mu \mathrm{m}$ DA suppressed the IRKC amplitude by $33 \pm 4 \%(n=5)$. Cnl, Control.
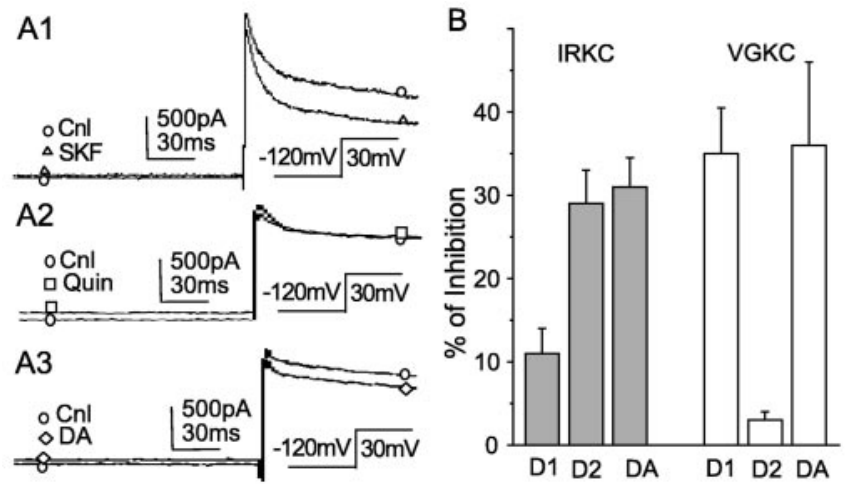

Figure 4. Functional differentiation of DA receptors. A modified protocol, as shown in the inset, included a step to $-120 \mathrm{mV}$ from the holding potential $-50 \mathrm{mV}$ and after a step to +30 $\mathrm{mV}$. This protocol elicited both IRKC and VGKC from a single neuron. $A 1$, Activation of $\mathrm{D}_{1} \mathrm{Rs}[0.1$ $\mu$ M SKF 81297 (SKF)] suppresses both VGKC and IRKC. The suppression of VGKC is substantial, but that of IRKC is relatively slight $(n=4)$. Note that the rapid inactivating component of VGKC $\left(I_{A}\right)$ in this neuron was not subject to the $D_{1} R$-mediated modulation. $A 2$, Activation of $D_{2}$ Rs with $0.1 \mu \mathrm{M}$ quinpirole (Quin) substantially suppressed IRKC but did not alter VGKC $(n=4)$. $A 3$, Perfusion of $20 \mu \mathrm{m}$ DA substantially suppressed both IRKC and VGKC $(n=3)$. B, The DA modulation of IRKC and VGKC is summarized. The IRKC amplitude is suppressed by perfusion of 0.1 $\mu \mathrm{M}$ SKF $81292(11 \pm 3 \% ; n=4)$, perfusion of $0.1 \mu \mathrm{m}$ quinpirole $(29 \pm 6 \% ; n=4)$, and perfusion of $20 \mu \mathrm{MDA}(31 \pm 4 \% ; n=3)$. The VGKC amplitude is suppressed by perfusion of $0.1 \mu \mathrm{MSKF} 81297(34 \pm 5 \% ; n=4)$ and $20 \mu \mathrm{M} \mathrm{DA}(35 \pm 8 \% ; n=3)$ but does not respond to $D_{2}$ R activation (net change of VGKC amplitude, $2 \pm 1 \% ; n=4$ ). Cnl, Control.
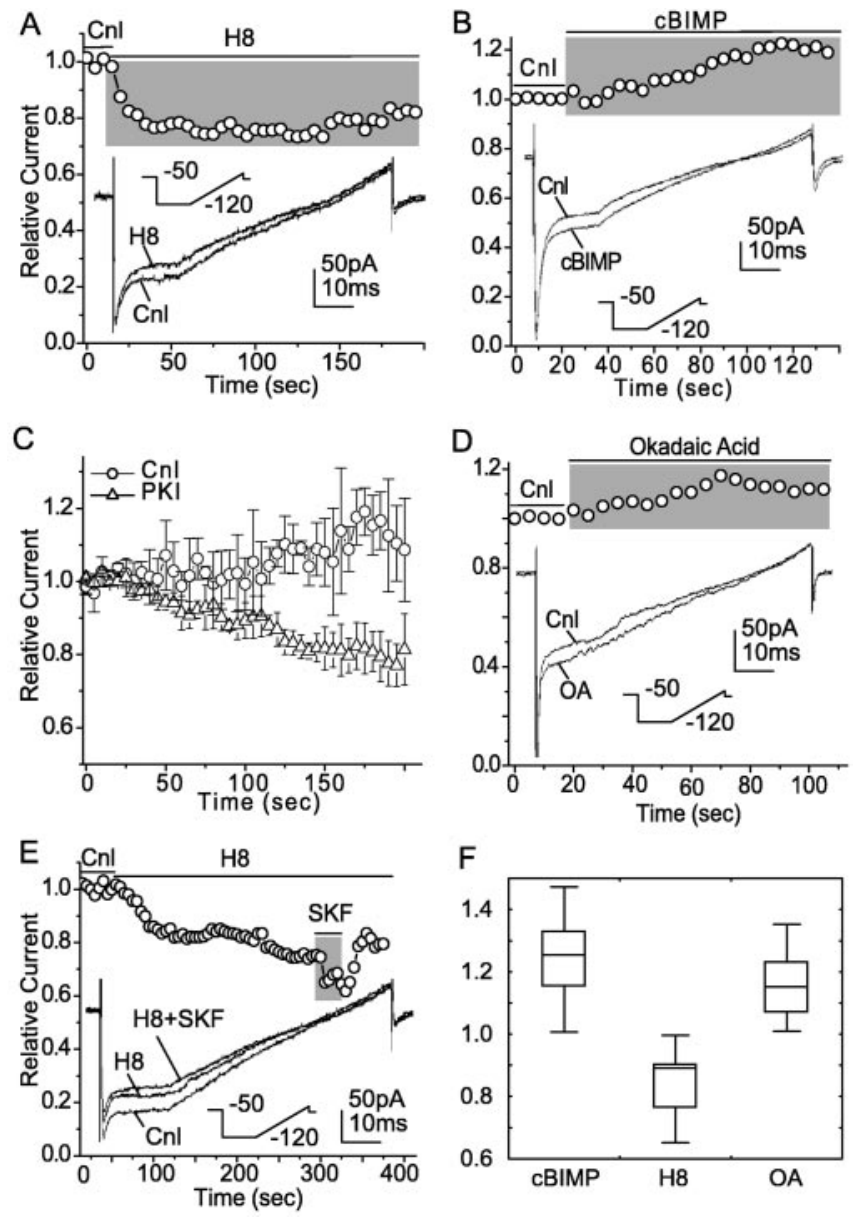

Figure 5. Modulation of IRKC by the CAMP-PKA signaling pathway. Inhibition of PKA activity with $\mathrm{H} 8(20 \mu \mathrm{M})$ suppressed the IRKC amplitude $(A)$, whereas stimulation of PKA activity with cBIMP $(25 \mu \mathrm{M})$ enhanced IRKC amplitude $(B)$. C, Intracellular dialysis of the PKA inhibitor PKI ( $1 \mathrm{U} / \mathrm{ml}$ in pipette solution) into the cell inhibited IRKC amplitude $(20 \pm 4 \% ; n=4) 3 \mathrm{~min}$ after rupture of the membrane. $D$, Perfusion of the protein phosphatase inhibitor okadaic acid (10 $\mu$ M) enhanced IRKC amplitude. E, Inhibition of basal PKA activities (20 $\mu \mathrm{m}$ H8) suppressed the amplitude of IRKC. Further activation of $D_{1} R[0.1 \mu \mathrm{M}$ SKF 81297 (SKF)] induced an additive inhibition, suggesting a PKA-independent mechanism underlying the $D_{1} R$-mediated inhibition of IRKC. F, Summary of the modulation of IRKC by the CAMP-PKA pathway. IRKC was enhanced by $23 \pm 7 \%(n=5)$ in the presence of CBIMP $(25 \mu \mathrm{M})$ and by $15 \pm 6 \%(n=5)$ when tested with okadaic acid $(10 \mu \mathrm{M})$. In contrast, $\mathrm{H} 8(20 \mu \mathrm{M})$ suppressed the amplitude of IRKC by $17 \pm$ $6 \%(n=5)$. Cnl, Control.

(Fig. 7C). Direct stimulation of adenylyl cyclase with $20 \mu \mathrm{M}$ forskolin, which stimulates the synthesis of cAMP, also induced bidirectional modulation of IRKC (Fig. 6C).

The above results argue that a slight stimulation of $D_{1}$ Rs $(0.1$ $\mu \mathrm{M}$ agonist) induces inhibition of IRKC, whereas a higher level of stimulation triggers activation of more than one modulatory pathway. Modulation of IRKC by $\mathrm{D}_{1} \mathrm{R}$ signaling diverges at the level of cAMP. The PKA-mediated phosphorylation-dependent pathway has been well demonstrated in our above experiments. We propose that the phosphorylation-independent pathway is mediated by a more direct interaction between IRK channels and cAMP. To examine this hypothesis, we used the outside-out patch technique, which primarily excludes coupling of cytosolic signaling molecules. Using this method, we measured a $\mathrm{Cs}^{+}$sensitive macroscopic IRKC from the outside-out patch (Fig. $7 A)$. Perfusion of the patch with $100 \mu \mathrm{M}$ Sp-8-bromo-cAMP induced a substantial inhibition of the macroscopic IRKC ( $25 \pm$ $7 \% ; n=5 ; p<0.05)$ (Fig. $7 B, C)$, suggesting a direct modulation 

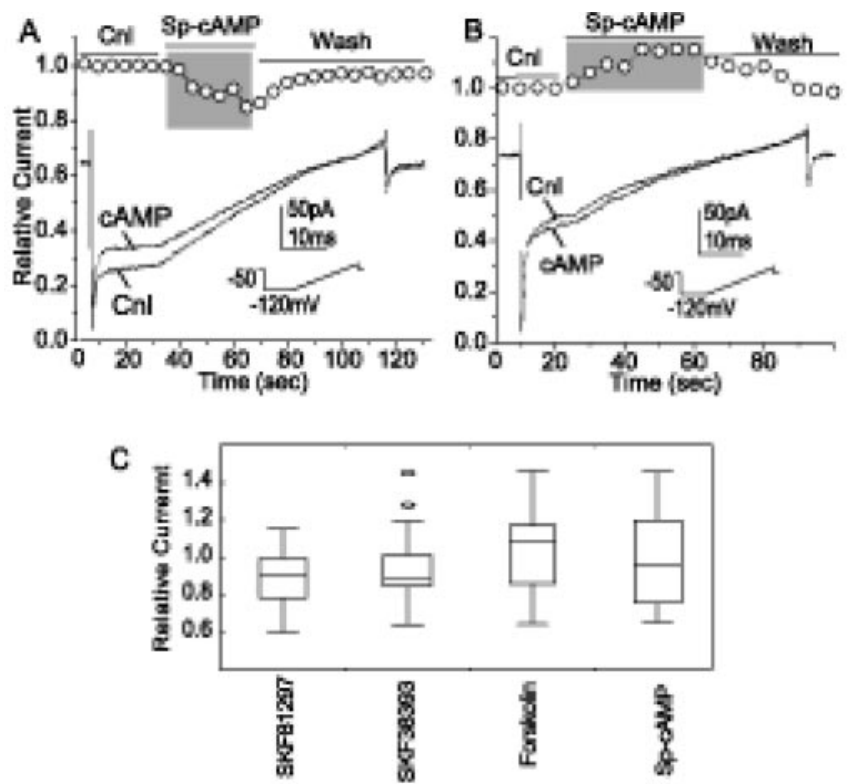

Figure 6. Modulation of IRKC by upstream components of the CAMP-PKA pathway. Perfusion of Sp-CAMP $(100 \mu \mathrm{M})$ inhibits IRKC amplitude in some neurons $(A)$ but enhances it in others (B).C, A summary shows that modulation of IRKC by $D_{1} R$ signaling diverges at the level of CAMP. When $D_{1} R$ was stimulated with a relatively higher intensity ( $5 \mu \mathrm{m}$ SKF81297), the IRKC amplitude was suppressed $>15 \%$ in 14 of 28 recorded neurons, whereas 4 of 28 recorded neurons displayed enhanced IRKC (enhancement, $>15 \%$ ), and the additional 10 neurons did not display obvious alterations (changes within 15\%). Similar responses were observed when the neurons were perfused with the partial $D_{1} R$ agonist SKF $38393(5 \mu \mathrm{M})$. IRKC was suppressed $>15 \%$ in 7 of 20 neurons, whereas in 5 of 20 recorded neurons, exhibited enhanced IRKC $(>15 \%)$. No obvious alterations (change, $<15 \%$ ) were observed in the other eight neurons. Perfusion of forskolin $(20 \mu \mathrm{M})$ induced similar divergent modulation of IRKC, suppression $(>15 \%)$ in 6 of 15 recorded neurons, enhancement ( $>15 \%)$ in 4 of 15 neurons, and no obvious alterations (change, $<15 \%$ ) in the other five neurons. Perfusion of $100 \mu \mathrm{m}$ Sp-CAMP also induces divergent modulation of IRKC: suppression (15\%) in 5 of 14 neurons, enhancement $(>15 \%)$ in 5 of 14 neurons, and no obvious alterations (change, $<15 \%$ ) in the other four neurons. Cnl, Control; Wash, washout.

of IRKC by cAMP. Perfusion of another membrane-permeable cyclic nucleotide, Rp-cAMP (100 $\mu \mathrm{M})$, also suppressed the macroscopic IRKC $(13 \pm 6 \% ; n=5)$ (Fig. $7 C)$. In contrast, the direct PKA stimulator cBIMP $(25 \mu \mathrm{M})$ and the PKA blocker H8 $(20 \mu \mathrm{M})$ failed to alter the macroscopic IRKC in outside-out patches, indicating that PKA-mediated phosphorylation was most likely disconnected from functional IRK channels in this preparation. This evidence argues a direct interaction between cAMP and IRK channels underling the inhibitory modulation by $\mathrm{D}_{1} \mathrm{Rs}$.

\section{Discussion}

These experiments sought to characterize how DA modulates IRKC, a critical conductance responsible for neuronal excitability, in deep layer (V and VI) mPFC pyramidal neurons and to identify the mechanisms responsible for such modulation. Intracellular recordings of cortical pyramidal neurons conducted in vivo indicate that these neurons fluctuate between hyperpolarized resting membrane potentials ("down-states") and plateau depolarizations ("up-states") (Cowan et al., 1994), facilitates the transition to the up-states, and helps to maintain that state. The present results, when combined with our previous findings (Dong and White, 2003), indicate that DA may modulate these membrane states by selectively modulating $\mathrm{K}^{+}$currents. Acting through both $\mathrm{D}_{1}$ and $\mathrm{D}_{2}$ class receptors, DA opposes IRKC to facilitate the transition to the up-state, whereas by suppressing the D-type VGKC through $\mathrm{D}_{1} \mathrm{R}$ stimulation, $\mathrm{DA}$ is able to sustain the up-state once achieved. Mechanistically, our findings indicate that $\mathrm{D}_{2} \mathrm{R}$ modulation occurs through inhibition of the adenylyl cyclase-cAMP-PKA signaling cascade, but $\mathrm{D}_{1} \mathrm{R}$ modulation surprisingly emerges through a direct interaction of cAMP with IRK channels.

\section{Characterization of isolated IRKC}

Although mPFC pyramidal neurons are known to exhibit IRKC (Yang et al., 1996), this is the first direct study of isolated IRKC. These currents exhibited strong inward rectification, were blocked by $\mathrm{Cs}^{+}$, and showed high selectivity for $\mathrm{K}^{+}$ions. These observations are consistent with known electrophysiological properties of IRKC (for review, see Jan and Jan, 1997). In addition to IRKC, there are at least three VGKCs $\left(I_{A}, I_{D}\right.$, and $\left.I_{K}\right)$ in mPFC pyramidal neurons, which may involve four types of channels (Dong and White, 2003). The three currents start to activate at approximately $-40 \mathrm{mV}$. Holding the membrane potential at $-50 \mathrm{mV}$ and stepping to more hyperpolarized voltages allowed us to isolate IRKC without detectable activation of VGKCs. Any contribution of G-protein-coupled IRK channels (GIRKs) to our recordings is unlikely for two reasons. First, the IRKC in the mPFC neurons exhibited relatively fast activation and inactivation kinetics, which is unlikely attributable to GIRK currents. Second, the IRKC was not affected by tertiapin $(200 \mathrm{~nm} ; n=4$; data not shown), an antagonist of Kir3 (GIRK1 and GIRK4) family subunits.

\section{DA modulation of IRKC}

DA plays a permissive role in maintaining normal activity of mPFC neurons through activation of the two DA receptor subfamilies, $D_{1} R$ and $D_{2} R$. The major finding of this study is that stimulation of either $\mathrm{D}_{1}$ Rs or $\mathrm{D}_{2}$ Rs produced inhibition of IRKC. Our results indicate that, although DA inhibits both IRKC and VGKC, VGKC is more sensitive to $D_{1} R$ modulation (Dong and White, 2003), whereas IRKC is more sensitive to $D_{2} R$ modulation. This functional differentiation provides the $\mathrm{DA}$ system with a dynamic ability to modulate mPFC pyramidal neurons. Kir channels (which conduct IRKC) and Kv channels (which conduct VGKC) differ in their subcellular locations, onsets of action during an excitable event, and contribution to neuronal excitability (Jan and Jan, 1997). DA $\mathrm{D}_{1^{-}}$and $\mathrm{D}_{2}$-class receptors also distribute unevenly with respect to subpopulations of PFC neurons, as can be discerned by the proportions of neurons responding to these agonists in our study, and with respect to subcellular loci within the same neuron (Goldman-Rakic, 1992; Ariano and Sibley, 1994). By taking advantage of differential coupling of its receptors with $\mathrm{K}^{+}$channels, DA can selectively modulate certain types of $\mathrm{K}^{+}$conductance at one time and at one location, spatially and temporally optimizing DA-mediated effects.

\section{Mechanisms of DA modulation}

DA $D_{1}$ - and $\mathrm{D}_{2}$-class receptors reciprocally couple to the adenylyl cyclase-cAMP-PKA signaling pathway (Stoof and Kebabian, 1984), yet in mPFC pyramidal neurons, they both suppress IRKC. Indeed, costimulation of these receptors by the endogenous agonist DA produced a suppression that appeared additive to that observed when the two different receptor classes were individually stimulated with selective agonists. To determine how $\mathrm{D}_{1}$ Rs and $\mathrm{D}_{2}$ Rs both decrease IRKC, we bypassed DA receptor modulation using agents that directly alter intracellular cAMP-PKA signaling. Given that $\mathrm{D}_{2} \mathrm{R}$ stimulation would be expected to decrease PKA activity, we directly reduced PKA activity with blocker H8 or PKI and observed a suppression of IRKC 

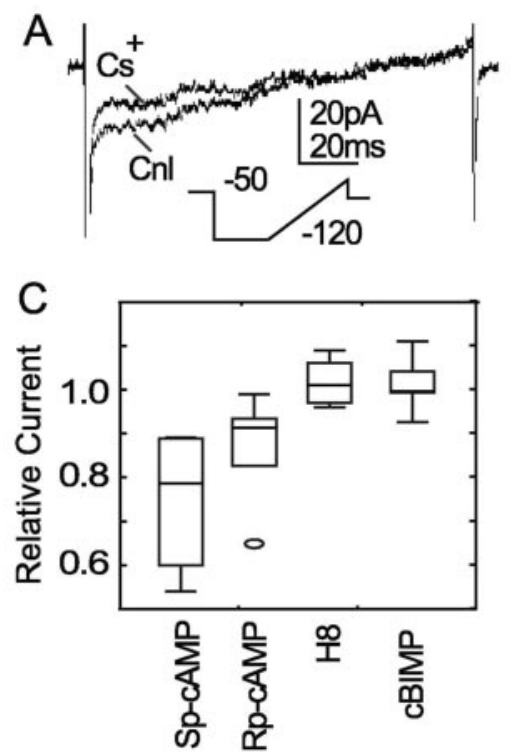

B

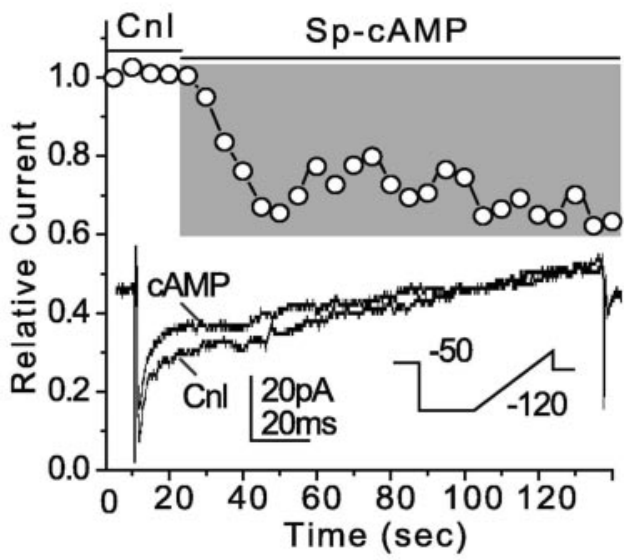

activity with $\mathrm{H} 8$, stimulation of $\mathrm{D}_{1}$ Rs with SKF 81297 induced an additive inhibition of IRKC, suggesting the existence of a PKA-independent modulation of IRKC by $\mathrm{D}_{1}$ Rs. So, if increasing PKA phosphorylation enhances IRKC, why did $D_{1} R$ stimulation produce the opposite effect?

We backed up from PKA modulation to study how cAMP, the immediate upstream component of this signaling cascade, would affect IRKC. Perfusion with the membrane-permeable cAMP analog Sp-cAMP or with forskolin, which stimulates the synthesis of cAMP, produced mixed effects, suppressing IRKC in 33\% of recorded cells but enhancing IRKC amplitude in a similar percentage. Thus, increasing intracellular cAMP, which would be triggered by $D_{1} R$ stimulation, modulates IRKC in opposite directions. This raises the question of why $D_{1} R$ stimulation did not result in such bidirectional modulation of IRKC. The answer appears to be a concentration-dependent effect. When we used higher concentrations of SKF 81297 or SKF $38393(5 \mu \mathrm{M})$, we increased the number of neurons that exhibited enhancement of IRKC. On the basis of these findings, we propose that the constitutive phosphorylation state of IRK channels is

amplitude comparable with that produced by the $\mathrm{D}_{2} \mathrm{R}$ agonist quinpirole. These results are consistent with $\mathrm{D}_{2} \mathrm{R}$ modulation occurring as a result of inhibition of adenylyl cyclase, leading to a reduction of cAMP activity and, thereby, constitutive PKA phosphorylation of IRK channels. Indeed, it has been suggested that normal functional activity of certain IRK channels (Kir2.1) requires PKA-mediated phosphorylation (Fakler et al., 1994). However, $\mathrm{D}_{2}$ Rs can also couple to other signaling systems, including activation of G-protein $\beta \gamma$ subunits leading to either membrane-delimited modulation of channels (Lledo et al., 1992; Yan et al., 1997) or activation of cytosolic signaling via phospholipase C (Yan et al., 1997; Hernández-López et al., 2000), possibilities that we have not directly excluded. Studies from nonnerve cell expression systems are controversial. For example, IRKC from transfected IRK1 (Kir2.1) homometrimers (in COS-7 cell lines) is inhibited by PKA-mediated phosphorylation (Wischmeyer and Karschin, 1996). In an endothelial cell line, however, transfected IRKC of the same subunits (Kir2.1) was shown to be insensitive to the activation of either PKA or PKC, or both (Kamouchi et al., 1997). In Xenopus oocytes, inhibition of transfected IRK3 (Kir2.3) currents is independent of phosphorylation (Chuang et al., 1997). Obviously, our observations in mPFC neurons are not consistent with all available data. Given that the intracellular regulatory networks in neurons are much more sophisticated than that in expression systems, this discrepancy may reflex the unique signaling pathway through which DA modulates the IRKC in mPFC neurons.

To examine the mechanisms responsible for $\mathrm{D}_{1} \mathrm{R}$ modulation of IRKC in mPFC pyramidal neurons, we began with experiments designed to directly modulate PKA. Stimulation of PKA with cBIMP enhanced IRKC amplitude, as did increasing phosphorylation of IRKC channels by blocking PP-induced dephosphorylation with okadaic acid. When we directly blocked PKA determined by an equilibrium between PKA and PP and that there is a PKA-independent mechanism by which $\mathrm{D}_{1} \mathrm{R}$ stimulation inhibits IRKC.

We next tested the possibility that the phosphorylationindependent pathway is mediated by a more direct interaction between IRK channels and cAMP. Such interactions have been observed previously in other cells. For example, $\beta$-adrenergic receptor-mediated modulation of IRKC has been proposed to occur through elevations of cAMP and direct binding to IRK channels by the nucleotide (Ito et al., 1997). Indeed, IRK channels are known to be directly modulated by small molecules, such as $\mathrm{Mg}^{2+}$, polyamines, ATP/ADP, $\mathrm{H}^{+}(\mathrm{pH})$, and nucleotides (for review, see Nichols and Lopatin, 1997). To determine whether cyclic nucleotides might directly suppress IRK channels in mPFC pyramidal neurons, we used the outside-out patch technique, which primarily excludes coupling of cytosolic signaling cascades. In this preparation, perfusion of the patch with the membrane-permeable cAMP analog Sp-cAMP suppressed the $\mathrm{Cs}^{+}$-sensitive macroscopic IRKC, as did Rp-cAMP, although less strongly. In contrast, neither stimulating PKA with cBIMP nor blocking PKA with $\mathrm{H} 8$ altered the macroscopic IRKC in outsideout patches, indicating that PKA-mediated phosphorylation of IRKC was no longer present in this preparation. This evidence argues for a direct interaction between cAMP and IRK channels underling the inhibitory modulation by $\mathrm{D}_{1}$ Rs. Modulation of IRKC by $D A D_{1} R$ and $D_{2} R$, as well as the differential modulation of VGKC and IRKC by DA, are summarized in Figure 8.

\section{Functional significance}

Pyramidal neurons in deep layers of mPFC integrate multiple excitatory and inhibitory inputs and send projections to many other brain areas. Through this network, the mPFC guides complex cognitive responses, such as working memory and the plan- 


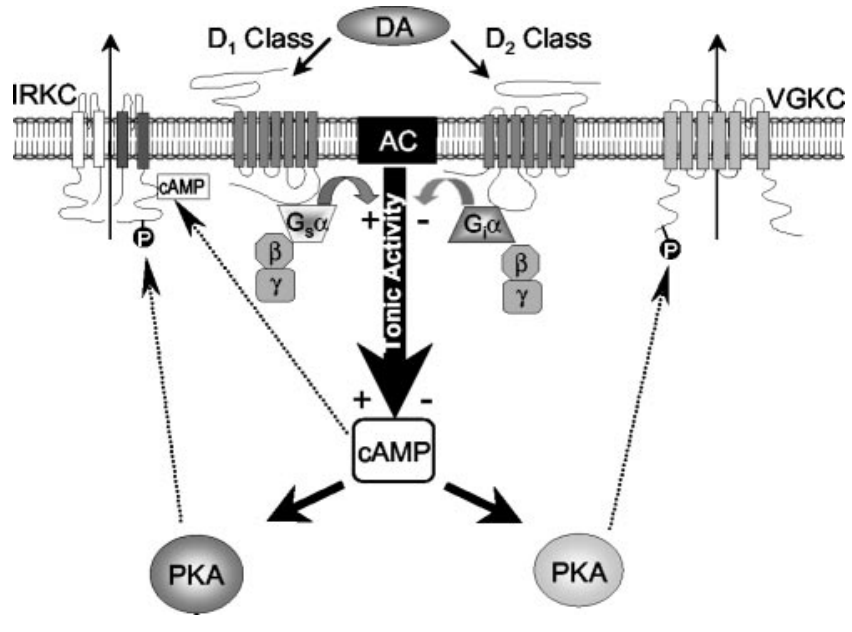

Figure 8. Dopamine modulates two types of $\mathrm{K}^{+}$currents in PFC neurons. DA modulates the VGKC and the IRKC via activation of DA $D_{1}$ - and $D_{2}$-class receptors. Activation of $D_{1} R$ increases intracellular cAMP level by activation of adenylyl cyclase (AC). The intracellular cAMP may diverge into two pathways: (1) direct interaction with IRK channels and reduction of IRKC; (2) PKA-dependent phosphorylation of IRK channels and enhancement of the IRKC. Although $D_{1} R-$ mediated modulation of IRKC is bidirectional, the inhibitory effect predominates under normal conditions. Activation of $D_{2} R$ inhibits the adenylyl cyclase activity, reduces basal activity of the CAMP-PKA signal transduction pathway, and dephosphorylates IRK channels, resulting in suppression of IRKC. DA modulates the VGKC primarily through $D_{1} R$, the activation of which stimulates the adenylyl cyclase-CAMP-PKA pathway and increases the phosphorylation of VGK channels, resulting in suppression of the D-type VGKC. P, Phosphorylation.

ning and execution of goal-directed behaviors (Goldman-Rakic, 1999b; Fuster, 2000). The excitatory state of mPFC pyramidal neurons directly affects reaction to various inputs and, consequently, its output to efferent nuclei. In the quiescent state, IRKC is one of the main determinants of input resistance, stabilizing the resting membrane potential toward $E_{\mathrm{K}}{ }^{+}$, thereby opposing excitatory input. In vivo electrophysiological studies indicate that PFC pyramidal neurons exhibit bi-states with a periodicity of $\sim 1$ $\mathrm{Hz}$ (Lavin and Grace, 2001). Given that DA, acting through both $\mathrm{D}_{1}$ Rs and $\mathrm{D}_{2} \mathrm{Rs}$, decreases IRKCs and, through $\mathrm{D}_{1} \mathrm{Rs}$, D-type VGKCs, it stands to reason that ambient extracellular DA should facilitate up-state transitions during excitatory drive but balance that effect via $\mathrm{D}_{1} \mathrm{R}$-mediated decreases in fast-inactivating $\mathrm{Na}^{+}$ current (Maurice et al., 2001). Along with modulation of both fast excitatory (NMDA) synapse (Law Tho et al., 1994; Lavin and Grace, 2001; Seamans et al., 2001) and inhibitory (GABA) synaptic currents (Penit-Soria et al., 1987; Gulledge and Jaffe, 2001), as well as undetermined effects on $\mathrm{Ca}^{+}$conductances, any changes in the basal activity of mesocortical DA neurons, or in the reactivity of DA receptors and transporters, would greatly influence the excitability and activity of the mPFC and thereby explain the exquisite sensitivity of this brain region to any changes in DA activity, as related to working memory and the organization of goal-directed behavior (Goldman-Rakic et al., 2000).

\section{References}

Ariano MA, Sibley DR (1994) Dopamine receptor distribution in the rat CNS: elucidation using anti-peptide antisera directed against $\mathrm{D}_{1 \mathrm{~A}}$ and $\mathrm{D}_{3}$ subtypes. Brain Res 649:95-110.

Chuang H, Jan YN, Jan LY (1997) Regulation of IRK3 inward rectifier K channel by $\mathrm{ml}$ acetylcholine receptor and intracellular magnesium. Cell 89:1121-1132.

Coetzee WA, Amarillo Y, Chiu J, Chow A, Lau D, McCormack T, Moreno H, Nadal MS, Ozaita A, Pountney D, Saganich M, Vega-Saenz dM, Rudy B (1999) Molecular diversity of $\mathrm{K}^{+}$channels. Ann NY Acad Sci 868: 233-285.
Cowan RL, Sesack SR, Van Bockstaele EJ, Branchereau P, Chain J, Pickel VM (1994) Analysis of synaptic inputs and targets of physiologically characterized neurons in rat frontal cortex: combined in vivo intracellular recording and immunolabeling. Synapse 17:101-114.

Dong Y, White FJ (2003) Dopamine $\mathrm{D}_{1}$-class receptors selectively modulate a slowly inactivating potassium current in rat medial prefrontal cortex pyramidal neurons. J Neurosci 23:2686-2695.

Fakler B, Brandle U, Glowatzki E, Zenner HP, Ruppersberg JP (1994) Kir2.1 inward rectifier $\mathrm{K}^{+}$channels are regulated independently by protein kinases and ATP hydrolysis. Neuron 13:1413-1420.

Fuster JM (2000) Prefrontal neurons in networks of executive memory. Brain Res Bull 52:331-336.

Fuster JM (2001) The prefrontal cortex — an update: time is of the essence. Neuron 30:319-333.

Goldman-Rakic PS (1992) Dopamine-mediated mechanisms of the prefrontal cortex. Semin Neurosci 4:149-159.

Goldman-Rakic PS (1999a) The "psychic" neuron of the cerebral cortex. Ann NY Acad Sci 868:13-26.

Goldman-Rakic PS (1999b) The physiological approach: functional architecture of working memory and disordered cognition in schizophrenia. Biol Psychiatry 46:650-661.

Gorelova NA, Yang CR (2000) Dopamine D1/D5 receptor activation modulates a persistent sodium current in rat prefrontal cortical neurons in vitro. J Neurophysiol 84:75-87.

Gulledge AT, Jaffe DB (2001) Multiple effects of dopamine on layer V pyramidal cell excitability in rat prefrontal cortex. J Neurophysiol 86:586-595

Hartman DS, Civelli O (1997) Dopamine receptor diversity: molecular and pharmacological perspectives. Prog Drug Res 48:173-194.

Hernández-López S, Tkatch T, Perez-Garci E, Galarraga E, Bargas J, Hamm $\mathrm{H}$, Surmeier J (2000) $\mathrm{D}_{2}$ dopamine receptors in striatal medium spiny neurons reduce L-type $\mathrm{Ca}^{2+}$ currents and excitability via a novel PLC $\beta 1$ $\mathrm{IP}_{3}$-calcineurin-signaling cascade. J Neurosci 20:8987-8995.

Hille B (2001) Ionic channels of excitable membranes. Ch 3.5.20. Sunderland, MA: Sinauer.

Hille B, Schwarz W (1978) Potassium channels as multi-ion single-file pores. J Gen Physiol 72:409-442.

Ito C, Onodera K, Watanabe T, Sato M (1997) Effects of histamine agents on methamphetamine-induced stereotyped behavior and behavioral sensitization in rats. Psychopharmacology (Berl) 130:362-367.

Jan LY, Jan YN (1997) Cloned potassium channels from eukaryotes and prokaryotes. Annu Rev Neurosci 20:91-123.

Kamouch M, van den Bremt K, Eggermont J, Droogmans G, Nilius B (1997) Modulation of inwardly rectifying potassium channels in cultured bovine pulmonary artery endothelial cells. J Physiol (Lond) 504:545-556.

Karschin C, Dissmann E, Stuhmer W, Karschin A (1996) IRK(1-3) and GIRK(1-4) inwardly rectifying $\mathrm{K}^{+}$channel mRNAs are differentially expressed in the adult rat brain. J Neurosci 16:3559-3570.

Knable MB, Weinberger DR (1997) Dopamine, the prefrontal cortex and schizophrenia. J Psychopharmacol 11:123-131.

Lavin A, Grace AA (2001) Stimulation of $\mathrm{D}_{1}$-type dopamine receptors enhances excitability in prefrontal cortical pyramidal neurons in a statedependent manner. Neuroscience 104:335-346.

Law-Tho D, Hirsch JC, Crepel F (1994) Dopamine modulation of synaptic transmission in rat prefrontal cortex: an in vitro electrophysiological study. Neurosci Res 21:151-160.

Lledo PM, Homburger V, Bockaert J, Vincent JD (1992) Differential G protein-mediated coupling of D2 dopamine receptors to $\mathrm{K}^{+}$and $\mathrm{Ca}^{2+}$ currents in rat anterior pituitary cells. Neuron 8:455-463.

Maurice N, Tkatch T, Meisler M, Sprunger LK, Surmeier DJ (2001) $D_{1} / D_{5}$ dopamine receptor activation differentially modulates rapidly inactivating and persistent sodium currents in prefrontal cortex pyramidal neurons. J Neurosci 21:2268-2277.

Mermelstein PG, Song WJ, Tkatch T, Yan Z, Surmeier DJ (1998) Inwardly rectifying potassium (IRK) currents are correlated with IRK subunit expression in rat nucleus accumbens medium spiny neurons. J Neurosci 18:6650-6661.

Miller EK, Cohen JD (2001) An integrated theory of prefrontal cortex function. Annu Rev Neurosci 24:167-202.

Nichols CG, Lopatin AN (1997) Inward rectifier potassium channels. Annu Rev Physiol 59:171-191.

Penit-Soria J, Audinat E, Crepel F (1987) Excitation of rat prefrontal cortical 
neurons by dopamine: an in vitro electrophysiological study. Brain Res 425:263-274.

Robbins TW, Everitt BJ (2002) Limbic-striatal memory systems and drug addiction. Neurobiol Learn Mem 78:625-636.

Rudy B, Kentros C, Weiser M, Fruhling D, Serodio P, Vega-Saenz dM, Ellisman MH, Pollock JA, Baker H (1992) Region-specific expression of a $\mathrm{K}^{+}$channel gene in brain. Proc Natl Acad Sci USA 89:4603-4607.

Seamans JK, Gorelova N, Durstewitz D, Yang CR (2001) Bidirectional dopamine modulation of GABAergic inhibition in prefrontal cortical pyramidal neurons. J Neurosci 21:3628-3638.

Serodio P, Rudy B (1998) Differential expression of Kv4 $\mathrm{K}^{+}$channel subunits mediating subthreshold transient $\mathrm{K}^{+}$(A-type) currents in rat brain. J Neurophysiol 79:1081-1091.

Sheng M, Tsaur ML, Jan YN, Jan LY (1994) Contrasting subcellular localization of the $\mathrm{Kv} 1.2 \mathrm{~K}^{+}$channel subunit in different neurons of rat brain. J Neurosci 14:2408-2417.

Stoof JC, Kebabian JW (1984) Two dopamine receptors: biochemistry, physiology and pharmacology. Life Sci 35:2281-2296.

Volkow ND, Fowler JS, Wang GJ, Goldstein RZ (2002) Role of dopamine, the frontal cortex and memory circuits in drug addiction: insight from imaging studies. Neurobiol Learn Mem 78:610-624.

Wang H, Kunkel DD, Schwartzkroin PA, Tempel BL (1994) Localization of Kv1.1 and Kv1.2, two K channel proteins, to synaptic terminals, somata, and dendrites in the mouse brain. J Neurosci 14:4588-4599.

Wischmeyer R, Karschin A (1996) Receptor stimulation causes slow inhibition of IRK1 inwardly rectifying $\mathrm{K}^{+}$channels by direct protein kinase A-mediated phosphorylation. Proc Natl Acad Sci USA 93:5819-5823.

Yan Z, Song WJ, Surmeier DJ (1997) $\mathrm{D}_{2}$ dopamine receptors reduce N-type $\mathrm{Ca}^{2+}$ currents in rat neostriatal cholinergic interneurons through a membrane-delimited, protein-kinase-C-insensitive pathway. J Neurophysiol 77:1003-1015.

Yang CR, Seamans JK (1996) Dopamine $\mathrm{D}_{1}$ receptor actions in layers V-VI rat prefrontal cortex neurons in vitro: modulation of dendritic-somatic signal integration. J Neurosci 16:1922-1935.

Yang CR, Seamans JK, Gorelova N (1999) Developing a neuronal model for the pathophysiology of schizophrenia based on the nature of electrophysiological actions of dopamine in the prefrontal cortex. Neuropsychopharmacology 21:161-194. 